\title{
Square-mean almost automorphic mild solutions to some stochastic differential equations in a Hilbert space
}

Yong-Kui Chang ${ }^{1 *}$, Zhi-Han Zhao ${ }^{1}$ and Gaston Mandata N'Guérékata ${ }^{2}$

\author{
* Correspondence: Izchangyk@163. \\ com \\ ${ }^{1}$ Department of Mathematics, \\ Lanzhou Jiaotong University, \\ Lanzhou, Gansu 730070, PR China \\ Full list of author information is \\ available at the end of the article
}

\author{
Abstract \\ This article deals primarily with the existence and uniqueness of square-mean almost \\ automorphic mild solutions for a class of stochastic differential equations in a real \\ separable Hilbert space. We study also some properties of square-mean almost \\ automorphic functions including a compostion theorem. To establish our main \\ results, we use the Banach contraction mapping principle and the techniques of \\ fractional powers of an operator. \\ Mathematics Subject Classification (2000) \\ 34K14, 60H10, 35B15, 34F05.
}

Keywords: Stochastic differential equations, Square-mean almost automorphic processes, Mild solutions

\section{Introduction}

In this article, we investigate the existence and uniqueness of square-mean almost automorphic solutions to the class of stochastic differential equations in the abstract form:

$$
d\left[x(t)-f\left(t, B_{1} x(t)\right)\right]=\left[A x(t)+g\left(t, B_{2} x(t)\right)\right] d t+h\left(t, B_{3} x(t)\right) d W(t), \quad t \in \mathbb{R},
$$

where $A: D(A) \subset L^{2}(\mathbb{P}, \mathbb{H}) \rightarrow L^{2}(\mathbb{P}, \mathbb{H})$ is the infinitesimal generator of an analytic semigroup of linear operators $\{T(t)\}_{t \geq 0}$ on $L^{2}(\mathbb{P}, \mathbb{H}), B_{i}, i=1,2,3$, are bounded linear operators that can be viewed as control terms, and $W(t)$ is a two-sided standard onedimensional Brownian motion defined on the filtered probability space $\left(\Omega, \mathcal{F}, \mathbb{P}, \mathcal{F}_{t}\right)$, where $\mathcal{F}_{t}=\sigma\{W(u)-W(v) ; u, v \leq t\}$. Here, $f, g$, and $h$ are appropriate functions to be specified later.

The concept of almost automorphy is an important generalization of the classical almost periodicity. They were introduced by Bochner [1,2]; for more details about this topic, we refer the reader to $[3,4]$. In recent years, the existence of almost periodic and almost automorphic solutions on different kinds of deterministic differential equations have been considerably investigated in lots of publications [5-15] because of its significance and applications in physics, mechanics, and mathematical biology.

Recently, the existence of almost periodic or pseudo almost periodic solutions to some stochastic differential equations have been considered in many publications, such

\section{SpringerOpen ${ }^{\circ}$}

(c) 2011 Chang et al; licensee Springer. This is an Open Access article distributed under the terms of the Creative Commons Attribution License (http://creativecommons.org/licenses/by/2.0), which permits unrestricted use, distribution, and reproduction in any medium, provided the original work is properly cited. 
as [16-22] and references therein. In a very recent article [23], the authors introduced a new concept of square-mean almost automorphic stochastic process. This paper generalizes the concept of quadratic mean almost periodic processes introduced by Bezandry and Diagana [18]. The authors established the existence and uniqueness of squaremean almost automorphic mild solutions to the following stochastic differential equations:

$$
\begin{gathered}
d x(t)=A x(t) d t+f(t) d t+W(\mathrm{t}) d W(t), \quad t \in \mathbb{R}, \\
d x(t)=A x(t) d t+f(t, x(t)) d t+g(t, x(t)) d W(t), \quad t \in \mathbb{R},
\end{gathered}
$$

in a Hilbert space $L^{2}(\mathbb{P}, \mathbb{H})$, where $\mathrm{A}$ is an infinitesimal generator of a $C_{0}$-semigroup $\{T(t)\}_{t} \geq 0$, and $W(t)$ is a two-sided standard one-dimensional Brown motion defined on the filtered probability space $\left(\Omega, \mathcal{F}, \mathbb{P}, \mathcal{F}_{t}\right)$, where $\mathcal{F}_{t}=\sigma\{W(u)-W(v) ; u, v \leq t\}$.

Motivated by the above mentioned studies [18,23], the main purpose of this article is to investigate the existence and uniqueness of square-mean almost automorphic solutions to the problem (1.1). Note that (1.1) is more general than the problem studied in [23]. We first use a sharper definition (Definition 2.1) of square-mean almost automorphic process than the Definition 2.5 in [23]. We then present some additional properties of square-mean almost automorphic processes (see Lemmas 2.4-2.5). Our main result is established by using fractional powers of linear operators and Banach contraction principle. The obtained result can be seen as a contribution to this emerging field since it improves and generalizes the results in [23].

The rest of this article is organized as follows. In section 2, we recall and prove some basic definitions, lemmas, and preliminary facts which will be used throughout this article. We also prove some additional properties of square-mean almost automorphic functions. In Section 3, we prove the existence and uniqueness of square-mean almost automorphic mild solutions to (1.1).

\section{Preliminaries}

In this section, we introduce some basic definitions, notations, lemmas, and technical results which are used in the sequel. For more details on this section, we refer the reader to $[23,24]$.

Throughout the article, we assume that $(\mathbb{H},\|\cdot\|\langle\cdot, \cdot\rangle)$ and $\left(\mathbb{K},\|\cdot\|_{\mathbb{K}},\langle\cdot, \cdot\rangle_{\mathbb{K}}\right)$ are two REAL separable Hilbert spaces. Let $(\Omega, \mathcal{F}, \mathbb{P})$ be a complete probability space. The notation $L^{2}(\mathbb{P}, \mathbb{H})$ stands for the space of all $\mathbb{H}$-valued random variables $x$ such that

$$
E\|x\|^{2}=\int_{\Omega}\|x\|^{2} d \mathbb{P}<\infty .
$$

For $x \in L^{2}(\mathbb{P}, \mathbb{H})$, let

$$
\|x\|_{2}=\left(\int_{\Omega}\|x\|^{2} d \mathbb{P}\right)^{\frac{1}{2}} .
$$

Then, it is routine to check that $L^{2}(\mathbb{P}, \mathbb{H})$ is a Hilbert space equipped with the norm $\|\cdot\|_{2}$. We let $L(\mathbb{K}, \mathbb{H})$ denote the space of all the linear-bounded operators from $\mathbb{K}$ into

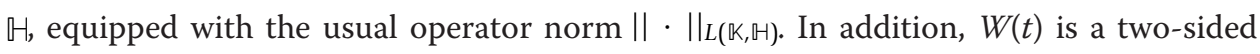
standard one-dimensional Brownian motion defined on the filtered probability space $\left(\Omega, \mathcal{F}, \mathbb{P}, \mathcal{F}_{t}\right)$, where $\mathcal{F}_{t}=\sigma\{W(u)-W(v) ; u, v \leq t\}$. 
Let $0 \in \rho(A)$ where $\rho(A)$ is the resolvent set of $A$; then, it is possible to define the fractional power $(-A)^{\alpha}$, for $0<\alpha \leq 1$, as a closed linear invertible operator on its domain $D\left((-A)^{\alpha}\right)$. Furthermore, the subspace $D\left((-A)^{\alpha}\right)$ is dense in $L^{2}(\mathbb{P}, \mathbb{H})$ and the expression

$$
\|x\|_{\alpha}=\left\|(-A)^{\alpha} x\right\|_{2}, \quad x \in D\left((-A)^{\alpha}\right),
$$

defines a norm on $D\left((-A)^{\alpha}\right)$. Hereafter, we denote by $L^{2}\left(\mathbb{P}, \mathbb{H}_{\alpha}\right)$ the Banach space $D$ $\left((-A)^{\alpha}\right)$ with norm $\|x\|_{\alpha}$.

The following properties hold by Pazy [25].

Lemma 2.1 Let $0<\gamma \leq \mu \leq 1$. Then, the following properties hold:

(i) $L^{2}\left(\mathbb{P}, \mathbb{H}_{\mu}\right)$ is a Banach space and $L^{2}\left(\mathbb{P}, \mathbb{H}_{\mu}\right) \hookrightarrow L^{2}\left(\mathbb{P}, \mathbb{H}_{\gamma}\right)$ is continuous.

(ii) The function $s \rightarrow(-A)^{\mu} T(s)$ is continuous in the uniform operator topology on (0,

$\infty)$, and there exists $M_{\mu}>0$ such that $\left\|(-\mathrm{A})^{\mu} \mathrm{T}(\mathrm{t})\right\| \leq \mathrm{M}_{\mu} \mathrm{e}^{-\delta \mathrm{t}^{-\mu}}$ for each $t>0$.

(iii) For each $x \in D\left((-A)^{\mu}\right)$ and $t \geq 0,(-A)^{\mu} T(t) x=T(t)(-A)^{\mu} x$.

(iv) $(-A)^{-\mu}$ is a bounded linear operator in $L^{2}(\mathbb{P}, \mathbb{H})$ with $D\left((-A)^{\mu}\right)=\operatorname{Im}\left(\left(-A^{)-\mu}\right)\right.$.

Definition $2.1([23])$ A stochastic process $x: \mathbb{R} \rightarrow L^{2}(\mathbb{P}, \mathbb{H})$ is said to be stochastically continuous if

$$
\lim _{t \rightarrow s} E\|x(t)-x(s)\|^{2}=0 .
$$

Definition 2.2 (compare with [23]) A stochastically continuous stochastic process $x: \mathbb{R} \rightarrow L^{2}(\mathbb{P}, \mathbb{H})$ is said to be square-mean almost automorphic if for every sequence of real numbers $\left\{s_{n}^{\prime}\right\}_{n \in \mathbb{N}}$, there exist a subsequence $\left\{s_{n}\right\}_{n \in \mathbb{N}}$ and a stochastic process

$$
\lim _{n \rightarrow \infty} E\left\|x\left(t+s_{n}\right)-y(t)\right\|^{2}=0 \text { and } \lim _{n \rightarrow \infty} E\left\|\gamma\left(t-s_{n}\right)-x(t)\right\|^{2}=0
$$

hold for each $t \in \mathbb{R}$.

The collection of all square-mean almost automorphic stochastic processes $x: \mathbb{R} \rightarrow L^{2}(\mathbb{P}, \mathbb{H})$ is denoted by $A A\left(\mathbb{R} ; L^{2}(\mathbb{P}, \mathbb{H})\right)$.

Lemma 2.2 ([23]) If $x, x_{1}$ and $x_{2}$ are all square-mean almost automorphic stochastic processes, then the following hold true:

(i) $x_{1}+x_{2}$ is square-mean almost automorphic.

(ii) $\lambda x$ is square-mean almost automorphic for every scalar $\lambda$.

(iii) There exists a constant $M>0$ such that $\sup _{t \in \mathbb{R}}\|x(t)\|_{2} \leq M$. That is, $x$ is bounded in $L^{2}(\mathbb{P}, \mathbb{H})$.

Lemma $2.3([23])\left(A A\left(\mathbb{R} ; L^{2}(\mathbb{P}, \mathbb{H})\right),\|\cdot\|_{\infty}\right)$ is a Banach space when it is equipped with the norm:

$$
\|x\|_{\infty}:=\sup _{t \in \mathbb{R}}\|x(t)\|_{2}=\sup _{t \in \mathbb{R}}\left(E\|x(t)\|^{2}\right)^{\frac{1}{2}},
$$

for $x \in A A\left(\mathbb{R} ; L^{2}(\mathbb{P}, \mathbb{H})\right)$. 
Let $L^{2}(\mathbb{P}, \tilde{H})$ be defined as $L^{2}(\mathbb{P}, \mathbb{H})$ and note that $L^{2}(\mathbb{P}, \mathbb{H}), L^{2}(\mathbb{P}, \tilde{H})$ are Banach spaces; then, we state the following lemmas (cf. $[3,13])$ :

Lemma 2.4 Let $f \in A A\left(\mathbb{R} ; L^{2}(\mathbb{P}, \mathbb{H})\right)$. Then, we have

$$
\begin{aligned}
& \text { (I) } h(t):=f(-t) \in A A\left(\mathbb{R} ; L^{2}(\mathbb{P}, \mathbb{H})\right) . \\
& \text { (II) } f_{a}(t):=f(t+a) \in A A\left(\mathbb{R} ; L^{2}(\mathbb{P}, \mathbb{H})\right) .
\end{aligned}
$$

Lemma 2.5 Let $\mathcal{L} \in L\left(L^{2}(\mathbb{P}, \tilde{\mathbb{H}}), L^{2}(\mathbb{P}, \mathbb{H})\right)$ and assume that $f \in A A\left(\mathbb{R} ; L^{2}(\mathbb{P}, \tilde{\mathbb{H}})\right)$. Then, $\mathcal{L} f \in A A\left(\mathbb{R} ; L^{2}(\mathbb{P}, \mathbb{H})\right)$.

Definition $2.3([23])$ A function $f: \mathbb{R} \times L^{2}(\mathbb{P}, \mathbb{H}) \rightarrow L^{2}(\mathbb{P}, \mathbb{H}),(t, x) \rightarrow f(t, x)$, which is jointly continuous, is said to be square-mean almost automorphic in $t \in \mathbb{R}$ for each $x \in L^{2}(\mathbb{P}, \mathbb{H})$ if for every sequence of real numbers $\left\{s_{n}^{\prime}\right\}_{n \in \mathbb{N}}$, there exist a subsequence $\left\{s_{n}\right\}$ $n \in \mathbb{N}$ and a stochastic process $\tilde{f}: \mathbb{R} \times L^{2}(\mathbb{P}, \mathbb{H}) \rightarrow L^{2}(\mathbb{P}, \mathbb{H})$ such that

$$
\lim _{n \rightarrow \infty} E\left\|f\left(t+s_{n}, x\right)-\tilde{f}(t, x)\right\|^{2}=0 \text { and } \lim _{n \rightarrow \infty} E\left\|\tilde{f}\left(t+s_{n}, x\right)-f(t, x)\right\|^{2}=0
$$

for each $t \in \mathbb{R}$ and each $x \in L^{2}(\mathbb{P}, \mathbb{H})$.

Theorem 2.1 ([23]) Let $f: \mathbb{R} \times L^{2}(\mathbb{P}, \mathbb{H}) \rightarrow L^{2}(\mathbb{P}, \mathbb{H}),(t, x) \rightarrow f(t, x)$ be square-mean almost automorphic in $t \in \mathbb{R}$ for each $x \in L^{2}(\mathbb{P}, \mathbb{H})$, and assume that $f$ satisfies Lipschitz condition in the following sense:

$$
E\|f(t, x)-f(t, y)\|^{2} \leq \tilde{M} E\|x-y\|^{2}
$$

for all $x, y \in L^{2}(\mathbb{P}, \mathbb{H})$ and for each $\mathrm{t} \in \mathbb{R}$, where $\tilde{M}>0$ is independent of $t$. Then, for any square-mean almost automorphic process $x: \mathbb{R} \rightarrow L^{2}(\mathbb{P}, \mathbb{H})$, the stochastic process $F: \mathbb{R} \rightarrow L^{2}(\mathbb{P}, \mathbb{H})$ given by $F(t)=f(t, x(t))$ is square-mean almost automorphic.

Definition 2.4 An $\mathcal{F}_{t}$-progressively measurable stochastic process $\{x(t)\}_{t \in \mathbb{R}}$ is called a mild solution of problem (1.1) on $R$ if the function $s \rightarrow A T(t-s) f\left(s, B_{1} x(s)\right)$ is integrable on $(-\infty, t)$ for each $t \in \mathbb{R}$, and $x(t)$ satisfies the corresponding stochastic integral equation

$$
\begin{aligned}
x(t)= & T(t-a)\left[x(a)-f\left(a, B_{1} x(a)\right)\right]+f\left(t, B_{1} x(t)\right)+\int_{a}^{t} A T(t-s) f\left(s, B_{1} x(s)\right) d s \\
& +\int_{a}^{t} T(t-s) g\left(s, B_{2} x(s)\right) d s+\int_{a}^{t} T(t-s) h\left(s, B_{3} x(s)\right) d W(s)
\end{aligned}
$$

for all $t \geq a$ and for each $a \in \mathbb{R}$.

\section{Main results}

In this section, we investigate the existence of a square-mean almost automorphic solution for the problem (1.1). We first list the following basic assumptions:

(H1) The operator $A: D(A) \subset L^{2}(\mathbb{P}, \mathbb{H}) \rightarrow L^{2}(\mathbb{P}, \mathbb{H})$ is the infinitesimal generator of an analytic semigroup of linear operators $\{T(t)\}_{t \geq 0}$ on $L^{2}(\mathbb{P}, \mathbb{H})$ and $M, \delta$ are positive numbers such that $\|T(t)\| \leq M e^{-\delta t}$ for $t \geq 0$.

(H2) The operators $B_{i}: L^{2}\left(\mathbb{P}, \mathbb{H}_{\alpha}\right) \rightarrow L^{2}(\mathbb{P}, \mathbb{H})$ for $i=1,2,3$, are bounded linear operators and $\varpi:=\max _{i=1,2,3}\left\{\left\|B_{i}\right\|_{L\left(L^{2}\left(\mathbb{P}, H_{\alpha}\right), L^{2}(\mathbb{P}, \mathbb{H})\right)}\right\}$.

(H3) There exists a positive number $\beta \in(0,1)$ such that $f: \mathbb{R} \times L^{2}(\mathbb{P}, \mathbb{H}) \rightarrow L^{2}\left(\mathbb{P}, \mathbb{H}_{\beta}\right)$ is square-mean almost automorphic in $t \in \mathbb{R}$ for each $\varphi \in L^{2}(\mathbb{P}, \mathbb{H})$. Let $L_{f}>0$ be such that for each $(t, \varphi),(t, \psi) \in \mathbb{R} \times L^{2}(\mathbb{P}, \mathbb{H})$ 


$$
E\left\|(-A)^{\beta} f(t, \varphi)-(-A)^{\beta} f(t, \psi)\right\|^{2} \leq L_{f} E\|\varphi-\psi\|^{2} .
$$

(H4) The functions $g: \mathbb{R} \times L^{2}(\mathbb{P}, \mathbb{H}) \rightarrow L^{2}(\mathbb{P}, \mathbb{H})$ and $h: \mathbb{R} \times L^{2}(\mathbb{P}, \mathbb{H}) \rightarrow L^{2}(\mathbb{P}, \mathbb{H})$ are square-mean almost automorphic in $t \in \mathbb{R}$ for each $\varphi \in L^{2}(\mathbb{P}, \mathbb{H})$. Moreover, $g$ and $h$ satisfy Lipschitz conditions in $\phi$ uniformly for $t$, that is, there exist positive numbers $L_{g}$, and $L_{h}$ such that

$$
E\|g(t, \varphi)-g(t, \psi)\|^{2} \leq L_{g} E\|\varphi-\psi\|^{2}
$$

and

$$
E\|h(t, \varphi)-h(t, \psi)\|^{2} \leq L_{g} E\|\varphi-\psi\|^{2}
$$

for all $t \in \mathbb{R}$ and each $\phi, \psi \in L^{2}(\mathbb{P}, \mathbb{H})$.

Theorem 3.1 Let $\alpha \in\left(0, \frac{1}{2}\right)$ and $\alpha<\beta<1$. If the conditions (H1)-(H4) are satisfied, then the problem (1.1) has a unique square-mean almost automorphic mild solution $x(\cdot) \in A A\left(\mathbb{R} ; L^{2}\left(\mathbb{P}, \mathbb{H}_{\alpha}\right)\right)$ provided that

$$
\begin{aligned}
L_{0}= & 4 \varpi^{2}\left\{\left\|(-A)^{\alpha-\beta}\right\|^{2} L_{f}+M_{1-\beta+\alpha}^{2} \delta^{2(\alpha-\beta)}[\Gamma(\beta-\alpha)]^{2} L_{f}+M_{\alpha}^{2} \delta^{2(\alpha-1)}[\Gamma(1-\alpha)]^{2} L_{g}(3.1)\right. \\
& \left.+M_{\alpha}^{2} L_{h}(2 \delta)^{2 \alpha-1} \Gamma(1-2 \alpha)\right\}<1,
\end{aligned}
$$

where $\Gamma(\cdot)$ is the gamma function.

Proof: Let $\Lambda: A A\left(\mathbb{R} ; L^{2}\left(\mathbb{P}, \mathbb{H}_{\alpha}\right)\right) \rightarrow A A\left(\mathbb{R} ; L^{2}\left(\mathbb{P}, \mathbb{H}_{\alpha}\right)\right)$ be the operator defined by

$$
\begin{aligned}
\Lambda x(t)= & f\left(t, B_{1} x(t)\right)+\int_{-\infty}^{t} A T(t-s) f\left(s, B_{1} x(s)\right) d s \\
& +\int_{-\infty}^{t} T(t-s) g\left(s, B_{2} x(s)\right) d s+\int_{-\infty}^{t} T(t-s) h\left(s, B_{3} x(s)\right) d W(s), \quad t \in \mathbb{R} .
\end{aligned}
$$

First, we prove that $\Lambda x$ is well defined. Indeed, let $x \in A A\left(\mathbb{R} ; L^{2}\left(\mathbb{P}, \mathbb{H}_{\alpha}\right)\right)$, then $s \rightarrow$ $B_{i} x(s)$ is in $A A\left(\mathbb{R} ; L^{2}(\mathbb{P}, \mathbb{H})\right)$ as $B_{i} \in L\left(L^{2}\left(\mathbb{P}, \mathbb{H}_{\alpha}\right), L^{2}(\mathbb{P}, \mathbb{H}), i=1,2,3\right.$ in virtue of Lemma 2.5, and hence, by Theorem 2.1, the function $s \rightarrow(-A)^{\beta} f\left(s, B_{1} x(s)\right)$ belongs to $A A\left(\mathbb{R} ; L^{2}(\mathbb{P}, \mathbb{H})\right)$ whenever $B_{1} x \in A A\left(\mathbb{R} ; L^{2}(\mathbb{P}, \mathbb{H})\right)$. Thus, using Lemma 2.2 (iii), it follows that there exists a constant $N_{f}>0$ such that $\sup _{t \in \mathbb{R}} E\left\|(-A)^{\beta} f\left(t, B_{1} x(t)\right)\right\|^{2} \leq N_{f}$. Moreover, from the continuity of $s \rightarrow A T(t-s)$ and $s \rightarrow T(t-s)$ in the uniform operator topology on $(-\infty, t)$ for each $t \in \mathbb{R}$ and the estimate

$$
\begin{aligned}
& E\left\|\int_{-\infty}^{t} A T(t-s) f\left(s, B_{1} x(s)\right) d s\right\|_{\alpha}^{2} \\
\leq & E\left(\int_{-\infty}^{t}\left\|(-A)^{1-\beta+\alpha} T(t-s)(-A)^{\beta} f\left(s, B_{1} x(s)\right)\right\| d s\right)^{2} \\
\leq & M_{1-\beta+\alpha}^{2} E\left(\int_{-\infty}^{t} e^{-\delta(t-s)}(t-s)^{\beta-\alpha-1}\left\|(-A)^{\beta} f\left(s, B_{1} x(s)\right)\right\| d s\right)^{2} \\
\leq & M_{1-\beta+\alpha}^{2}\left(\int_{-\infty}^{t} e^{-\delta(t-s)}(t-s)^{\beta-\alpha-1} d s\right) \\
& \times\left(\int_{-\infty}^{t} e^{-\delta(t-s)}(t-s)^{\beta-\alpha-1} E\left\|(-A)^{\beta} f\left(s, B_{1} x(s)\right)\right\|^{2} d s\right) \\
\leq & M_{1-\beta+\alpha}^{2}\left(\int_{-\infty}^{t} e^{-\delta(t-s)}(t-s)^{\beta-\alpha-1} d s\right)^{2} \sup _{t \in \mathbb{R}} E\left\|(-A)^{\beta} f\left(t, B_{1} x(t)\right)\right\|^{2} \\
\leq & M_{1-\beta+\alpha}^{2} N_{f} \delta^{2(\alpha-\beta)}[\Gamma(\beta-\alpha)]^{2},
\end{aligned}
$$


it follows that $s \rightarrow A T(t-s) f\left(s, B_{1} x(s)\right), s \rightarrow T(t-s) g\left(s, B_{2} x(s)\right)$ and $s \rightarrow T(t-s) h(s$, $\left.B_{3} x(s)\right)$ are integrable on $(-\infty, t)$ for every $t \in \mathbb{R}$, therefore, $\Lambda x$ is well defined.

Next, we show that $\Lambda x(t) \in A A\left(\mathbb{R} ; L^{2}\left(\mathbb{P}, \uplus_{\alpha}\right)\right)$. Let us consider the nonlinear operator $\Lambda_{1} x, \Lambda_{2} x$, and $\Lambda_{3} x$ acting on the Banach space $A A\left(\mathbb{R} ; L^{2}\left(\mathbb{P}, \mathbb{H}_{\alpha}\right)\right)$ defined by

$$
\begin{aligned}
& \Lambda_{1} x(t)=\int_{-\infty}^{t} A T(t-s) f\left(s, B_{1} x(s)\right) d s, \\
& \Lambda_{2} x(t)=\int_{-\infty}^{t} T(t-s) g\left(s, B_{2} x(s)\right) d s
\end{aligned}
$$

and

$$
\Lambda_{3} x(t)=\int_{-\infty}^{t} T(t-s) h\left(s, B_{2} x(s)\right) d W(s)
$$

respectively. Now, let us prove that $\Lambda_{1} x(t) \in A A\left(\mathbb{R} ; L^{2}\left(\mathbb{P}, \mathbb{H}_{\alpha}\right)\right)$. Let $\left\{s_{n}^{\prime}\right\}_{n \in \mathbb{N}}$ be an arbitrary sequence of real numbers. Since $F(\cdot)=(-A)^{\beta} f\left(\cdot, B_{1} x(\cdot)\right) \in A A\left(\mathbb{R} ; L^{2}(\mathbb{P}, \mathbb{H})\right)$, there exists a subsequence $\left\{s_{n}\right\}_{n \in \mathbb{N}}$ of $\left\{s_{n}^{\prime}\right\}_{n \in \mathbb{N}}$ such that for certain stochastic process $\tilde{F}$

$$
\lim _{n \rightarrow \infty} E\left\|F\left(t+s_{n}\right)-\tilde{F}(t)\right\|^{2}=0 \quad \text { and } \quad \lim _{n \rightarrow \infty} E\left\|\tilde{F}\left(t+s_{n}\right)-F(t)\right\|^{2}=0
$$

hold for each $t \in \mathbb{R}$. Moreover, if we let $\widetilde{\Lambda_{1}} x(t)=\int_{-\infty}^{t}(-A)^{1-\beta} T(t-s) \tilde{F}(s) d s$, then by using Cauchy-Schwarz inequality, we have

$$
\begin{aligned}
& E\left\|\Lambda_{1} x\left(t+s_{n}\right)-\widetilde{\Lambda_{1}} x(t)\right\|_{\alpha}^{2} \\
= & E\left\|\int_{-\infty}^{t+s_{n}} A T\left(t+s_{n}-s\right) f\left(s, B_{1} x(s)\right) d s-\int_{-\infty}^{t}(-A)^{1-\beta} T(t-s) \tilde{F}(s) d s\right\|_{\alpha}^{2} \\
= & E\left\|\int_{-\infty}^{t}(-A)^{1-\beta} T(t-s) F\left(s+s_{n}\right) d s-\int_{-\infty}^{t}(-A)^{1-\beta} T(t-s) \tilde{F}(s) d s\right\|_{\alpha}^{2} \\
\leq & E\left(\int_{-\infty}^{t}\left\|(-A)^{1-\beta+\alpha} T(t-s)\right\|\left\|F\left(s+s_{n}\right)-\tilde{F}(s)\right\| d s\right)^{2} \\
\leq & M_{1-\beta+\alpha}^{2} E\left(\int_{-\infty}^{t} e^{-\delta(t-s)}(t-s)^{\beta-\alpha-1}\left\|F\left(s+s_{n}\right)-\tilde{F}(s)\right\| d s\right)^{2} \\
\leq & M_{1-\beta+\alpha}^{2}\left(\int_{-\infty}^{t} e^{-\delta(t-s)}(t-s)^{\beta-\alpha-1} d s\right)^{t} \\
& \times\left(\int_{-\infty}^{t} e^{-\delta(t-s)}(t-s)^{\beta-\alpha-1} E\left\|F\left(s+s_{n}\right)-\tilde{F}(s)\right\|^{2} d s\right) \\
\leq & M_{1-\beta+\alpha}^{2}\left(\int_{-\infty}^{t} e^{-\delta(t-s)}(t-s)^{\beta-\alpha-1} d s\right)^{2} \sup _{t \in \mathbb{R}} E\left\|F\left(t+s_{n}\right)-\tilde{F}(t)\right\|^{2} \\
\leq & M_{1-\beta+\alpha}^{2} \delta^{2(\alpha-\beta)}[\Gamma(\beta-\alpha)]^{2} \sup _{t \in \mathbb{R}} E\left\|F\left(t+s_{n}\right)-\tilde{F}(t)\right\|^{2} .
\end{aligned}
$$

Thus, by (3.2), we immediately obtain that

$$
\lim _{n \rightarrow \infty} E\left\|\Lambda_{1} x\left(t+s_{n}\right)-\widetilde{\Lambda_{1}} x(t)\right\|_{\alpha}^{2}=0,
$$

for each $t \in \mathbb{R}$, and we can show in a similar way that

$$
\lim _{n \rightarrow \infty} E\left\|\widetilde{\Lambda_{1}} x\left(t-s_{n}\right)-\Lambda_{1} x(t)\right\|_{\alpha}^{2}=0,
$$


for each $t \in \mathbb{R}$. Thus, we conclude that $\Lambda_{1} x(t) \in A A\left(\mathbb{R} ; L^{2}\left(\mathbb{P}, \mathbb{H}_{\alpha}\right)\right)$.

Similarly, by using Theorem 2.1 , one easily sees that $s \rightarrow g\left(s, B_{2} x(s)\right)$ belongs to $A A\left(\mathbb{R} ; L^{2}(\mathbb{P}, \mathbb{H})\right)$ whenever $B_{2} x \in A A\left(\mathbb{R} ; L^{2}(\mathbb{P}, \mathbb{H})\right)$. Since $G(\cdot)=g\left(\cdot, B_{2} x(\cdot)\right) \in A A\left(\mathbb{R} ; L^{2}(\mathbb{P}, \mathbb{H})\right)$ for every sequence of real numbers $\left\{s_{n}^{\prime}\right\}_{n \in \mathbb{N}}$, there exists a subsequence $\left\{s_{n}\right\}_{n \in \mathbb{N}} \subset\left\{s_{n}^{\prime}\right\}_{n \in \mathbb{N}}$ such that for certain stochastic process $\tilde{G}$

$$
\lim _{n \rightarrow \infty} E\left\|G\left(t+s_{n}\right)-\tilde{G}(t)\right\|^{2}=0 \quad \text { and } \quad \lim _{n \rightarrow \infty} E\left\|\tilde{G}\left(t+s_{n}\right)-G(t)\right\|^{2}=0
$$

hold for each $t \in \mathbb{R}$. Moreover, if we let $\widetilde{\Lambda_{2}} x(t)=\int_{-\infty}^{t} T(t-s) \tilde{G}(s) d s$, then by using Cauchy-Schwarz inequality, we get

$$
\begin{aligned}
& E\left\|\Lambda_{2} x\left(t+s_{n}\right)-\widetilde{\Lambda_{2}} x(t)\right\|_{\alpha}^{2} \\
= & E\left\|\int_{-\infty}^{t+s_{n}} T\left(t+s_{n}-s\right) g\left(s, B_{2} x(s)\right) d s-\int_{-\infty}^{t} T(t-s) \tilde{G}(s) d s\right\|_{\alpha}^{2} \\
\leq & E\left(\int_{-\infty}^{t}\left\|(-A)^{\alpha} T(t-s)\left[G\left(s+s_{n}\right)-\tilde{G}(s)\right]\right\| d s\right)^{2} \\
\leq & M_{\alpha}^{2} E\left(\int_{-\infty}^{t} e^{-\delta(t-s)}(t-s)^{-\alpha}\left\|G\left(s+s_{n}\right)-\tilde{G}(s)\right\| d s\right)^{2} \\
\leq & M_{\alpha}^{2}\left(\int_{-\infty}^{t} e^{-\delta(t-s)}(t-s)^{-\alpha} d s\right)\left(\int_{-\infty}^{t} e^{-\delta(t-s)}(t-s)^{-\alpha} E\left\|G\left(s+s_{n}\right)-\tilde{G}(s)\right\|^{2} d s\right) \\
\leq & M_{\alpha}^{2}\left(\int_{-\infty}^{t} e^{-\delta(t-s)}(t-s)^{-\alpha} d s\right)^{2} \sup _{t \in \mathbb{R}} E\left\|G\left(t+s_{n}\right)-\tilde{G}(t)\right\|^{2} \\
\leq & M_{\alpha}^{2} \delta^{2(\alpha-1)}[\Gamma(1-\alpha)]^{2} \sup _{t \in \mathbb{R}} E\left\|G\left(t+s_{n}\right)-\tilde{G}(t)\right\|^{2} .
\end{aligned}
$$

Thus, by (3.3), we immediately obtain that

$$
\lim _{n \rightarrow \infty} E\left\|\Lambda_{2} x\left(t+s_{n}\right)-\widetilde{\Lambda_{2}} x(t)\right\|_{\alpha}^{2}=0
$$

for each $t \in \mathbb{R}$, and we can show in a similar way that

$$
\lim _{n \rightarrow \infty} E\left\|\widetilde{\Lambda_{2}} x\left(t+s_{n}\right)-\Lambda_{2} x(t)\right\|_{\alpha}^{2}=0,
$$

for each $t \in \mathbb{R}$. Thus, we conclude that $\Lambda_{2} x(t) \in A A\left(\mathbb{R} ; L^{2}\left(\mathbb{P}, \mathbb{H}_{\alpha}\right)\right)$.

Now, by using Theorem 2.1, one easily sees that $s \rightarrow h\left(s, B_{3} x(s)\right)$ is in $A A\left(\mathbb{R} ; L^{2}(\mathbb{P}, \mathbb{H})\right) \quad$ whenever $\quad B_{3} x(t) \in A A\left(\mathbb{R P} ; L^{2}(\mathbb{P}, \mathbb{H})\right)$. Since $H(\cdot)=h\left(\cdot, B_{2} x(\cdot)\right) \in A A\left(\mathbb{R} ; L^{2}(\mathbb{P}, \mathbb{H})\right)$, for every sequence of real numbers $\left\{s_{n}^{\prime}\right\}_{n \in \mathbb{N}}$, there exists a subsequence $\left\{s_{n}\right\}_{n \in \mathbb{N}} \subset\left\{s_{n}^{\prime}\right\}_{n \in \mathbb{N}}$ such that for certain stochastic process $\tilde{H}$

$$
\lim _{n \rightarrow \infty} E\left\|H\left(t+s_{n}\right)-\tilde{H}(t)\right\|^{2}=0 \quad \text { and } \quad \lim _{n \rightarrow \infty} E\left\|\tilde{H}\left(t+s_{n}\right)-H(t)\right\|^{2}=0
$$

hold for each $t \in \mathbb{R}$. The next step consists of showing that $\Lambda_{3} x(t) \in A A\left(\mathbb{R} ; L^{2}\left(\mathbb{P}, \mathbb{H}_{\alpha}\right)\right)$. Let $\widetilde{W}(\sigma):=W\left(\sigma+s_{n}\right)-W\left(s_{n}\right)$ for each $\sigma \in \mathbb{R}$. Note that $\widetilde{W}$ is also a Brownian motion and has the same distribution as $W$. Moreover, if we let $\widetilde{\Lambda_{3}} x(t)=\int_{-\infty}^{t} T(t-s) \tilde{H}(s) d W(s)$, then by making a change of variables $\sigma=s-s_{n}$ we get

$$
\begin{aligned}
& E\left\|\Lambda_{3} x\left(t+s_{n}\right)-\widetilde{\Lambda_{3}} x(t)\right\|_{\alpha}^{2} \\
= & E\left\|\int_{-\infty}^{t+s_{n}} T\left(t+s_{n}-s\right) H(s) d W(s)-\int_{-\infty}^{t} T(t-s) \tilde{H}(s) d W(s)\right\|_{\alpha}^{2} \\
= & E\left\|\int_{-\infty}^{t} T(t-\sigma)\left[H\left(\sigma+s_{n}\right)-\tilde{H}(\sigma)\right] d \tilde{W}(\sigma)\right\|_{\alpha}^{2}
\end{aligned}
$$


Thus, using an estimate on Ito integral established in Ichikawa [26], we obtain that

$$
\begin{aligned}
& E\left\|\Lambda_{3} x\left(t+s_{n}\right)-\widetilde{\Lambda_{3}} x(t)\right\|_{\alpha}^{2} \\
\leq & E\left(\int_{-\infty}^{t}\left\|(-A)^{\alpha} T(t-\sigma)\left[H\left(\sigma+s_{n}\right)-\tilde{H}(\sigma)\right]\right\|^{2} d s\right) \\
\leq & M_{\alpha}^{2} \int_{-\infty}^{t} e^{-2 \delta(t-s)}(t-s)^{-2 \alpha} E\left\|H\left(\sigma+s_{n}\right)-\tilde{H}(\sigma)\right\|^{2} d s \\
\leq & M_{\alpha}^{2}(2 \delta)^{2 \alpha-1} \Gamma(1-2 \alpha) \sup _{t \in \mathbb{R}} E\left\|H\left(t+s_{n}\right)-\tilde{H}(t)\right\|^{2} .
\end{aligned}
$$

Thus, by (3.4), we immediately obtain that

$$
\lim _{n \rightarrow \infty} E\left\|\Lambda_{3} x\left(t+s_{n}\right)-\widetilde{\Lambda_{3}} x(t)\right\|_{\alpha}^{2}=0,
$$

for each $t \in \mathbb{R}$. Arguing in a similar way, we infer that

$$
\lim _{n \rightarrow \infty} E\left\|\widetilde{\Lambda_{3}} x\left(t+s_{n}\right)-\Lambda_{3} x(t)\right\|_{\alpha}^{2}=0
$$

for each $t \in \mathbb{R}$. Thus, we conclude that $\Lambda_{3} x(t) \in A A\left(\mathbb{R} ; L^{2}\left(\mathbb{P}, \mathbb{H}_{\alpha}\right)\right)$. Since $f\left(\cdot, B_{1} x(\cdot)\right) \in A A\left(\mathbb{R} ; L^{2}\left(\mathbb{P}, \mathbb{H}_{\beta}\right)\right) \subset A A\left(\mathbb{R} ; L^{2}\left(\mathbb{P}, \mathbb{H}_{\alpha}\right)\right)$, and in view of the above, it is clear that $\Lambda$ maps $A A\left(\mathbb{R} ; L^{2}\left(\mathbb{P}, \mathbb{H}_{\alpha}\right)\right)$ into itself.

Now the remaining task is to prove that is a contraction mapping on $A A\left(\mathbb{R} ; L^{2}\left(\mathbb{P}, \mathbb{H}_{\alpha}\right)\right)$. Indeed, for each $t \in \mathbb{R}, x, y \in A A\left(\mathbb{R} ; L^{2}\left(\mathbb{P}, \mathbb{H}_{\alpha}\right)\right)$, we see that

$$
\begin{aligned}
& E\|(\Lambda x)(t)-(\Lambda y)(t)\|_{\alpha}^{2} \\
= & E \| f\left(t, B_{1} x(t)\right)-f\left(t, B_{1} \gamma(t)\right)+\int_{-\infty}^{t} A T(t-s)\left[f\left(s, B_{1} x(s)\right)-f\left(s, B_{1} \gamma(s)\right)\right] d s \\
& +\int_{-\infty}^{t} T(t-s)\left[g\left(s, B_{2} x(s)\right)-g\left(s, B_{2} y(s)\right)\right] d s \\
& +\int_{-\infty}^{t} T(t-s)\left[h\left(s, B_{3} x(s)\right)-h\left(s, B_{3} \gamma(s)\right)\right] d W(s) \|_{\alpha}^{2} \\
\leq & 4 E\left\|f\left(t, B_{1} x(t)\right)-f\left(t, B_{1} \gamma(t)\right)\right\|_{\alpha}^{2} \\
& +4 E\left\|\int_{-\infty}^{t} A T(t-s)\left[f\left(s, B_{1} x(s)\right)-f\left(s, B_{1} \gamma(s)\right)\right] d s\right\|_{\alpha}^{2} \\
& +4 E\left\|\int_{-\infty}^{t} T(t-s)\left[g\left(s, B_{2} x(s)\right)-g\left(s, B_{2} \gamma(s)\right)\right] d s\right\|_{\alpha}^{2} \\
& +4 E\left\|\int_{-\infty}^{t} T(t-s)\left[h\left(s, B_{3} x(s)\right)-h\left(s, B_{3} \gamma(s)\right)\right] d W(s)\right\|_{\alpha}^{2} \\
\leq & 4\left\|(-A)^{\alpha-\beta}\right\|^{2} E\left\|(-A)^{\beta} f\left(t, B_{1} x(t)\right)-(-A)^{\beta} f\left(t, B_{1} \gamma(t)\right)\right\|^{2} \\
& +4 E\left(\int_{-\infty}^{t}\left\|(-A)^{1-\beta+\alpha} T(t-s)\left[(-A)^{\beta} f\left(s, B_{1} x(s)\right)-(-A)^{\beta} f\left(s, B_{1} \gamma(s)\right)\right]\right\| d s\right)^{2} \\
& +4 E\left(\int_{-\infty}^{t}\left\|(-A)^{\alpha} T(t-s)\left[g\left(s, B_{2} x(s)\right)-g\left(s, B_{2} \gamma(s)\right)\right]\right\| d s\right)^{2} \\
& +4 E\left\|\int_{-\infty}^{t} T(t-s)\left[h\left(s, B_{3} x(s)\right)-h\left(s, B_{3} y(s)\right)\right] d W(s)\right\|_{\alpha}^{2} .
\end{aligned}
$$

We first evaluate the first term of the right-hand side as follows:

$$
\begin{aligned}
& 4\left\|(-A)^{\alpha-\beta}\right\|^{2} E\left\|(-A)^{\beta} f\left(t, B_{1} x(t)\right)-(-A)^{\beta} f\left(t, B_{1} y(t)\right)\right\|^{2} \\
\leq & 4\left\|(-A)^{\alpha-\beta}\right\|^{2} L_{f} E\left\|B_{1} x(t)-B_{1} \gamma(t)\right\|^{2} \\
\leq & 4\left\|(-A)^{\alpha-\beta}\right\|^{2} L_{f} \varpi^{2} \sup _{t \in \mathbb{R}} E\|x(t)-\gamma(t)\|_{\alpha}^{2} .
\end{aligned}
$$


As regards the second term, by Cauchy-Schwarz inequality, we have

$$
\begin{aligned}
& 4 E\left(\int_{-\infty}^{t}\left\|(-A)^{1-\beta+\alpha} T(t-s)\left[(-A)^{\beta} f\left(s, B_{1} x(s)\right)-(-A)^{\beta} f\left(s, B_{1} y(s)\right)\right]\right\| d s\right)^{2} \\
\leq & 4 M_{1-\beta+\alpha}^{2} E\left(e^{-\delta(t-s)}(t-s)^{\beta-\alpha-1}\left\|(-A)^{\beta} f\left(s, B_{1} x(s)\right)-(-A)^{\beta} f\left(s, B_{1} \gamma(s)\right)\right\| d s\right)^{2} \\
\leq & 4 M_{1-\beta+\alpha}^{2} E\left[\left(\int_{-\infty}^{t} e^{-\delta(t-s)}(t-s)^{\beta-\alpha-1} d s\right)\right. \\
& \left.\times\left(\int_{-\infty}^{t} e^{-\delta(t-s)}(t-s)^{\beta-\alpha-1}\left\|(-A)^{\beta} f\left(s, B_{1} x(s)\right)-(-A)^{\beta} f\left(s, B_{1} \gamma(s)\right)\right\|^{2} d s\right)\right] \\
\leq & 4 M_{1-\beta+\alpha}^{2}\left(\int_{-\infty}^{t} e^{-\delta(t-s)}(t-s)^{\beta-\alpha-1} d s\right) \\
& \times\left(\int_{-\infty}^{t} e^{-\delta(t-s)}(t-s)^{\beta-\alpha-1} E\left\|(-A)^{\beta} f\left(s, B_{1} x(s)\right)-(-A)^{\beta} f\left(s, B_{1} \gamma(s)\right)\right\|^{2} d s\right) \\
\leq & 4 M_{1-\beta+\alpha}^{2} L_{f}\left(\int_{-\infty}^{t} e^{-\delta(t-s)}(t-s)^{\beta-\alpha-1} d s\right) \\
& \times\left(\int_{-\infty}^{t} e^{-\delta(t-s)}(t-s)^{\beta-\alpha-1} E\left\|B_{1} x(s)-B_{1} \gamma(s)\right\|^{2} d s\right) \\
\leq & 4 M_{1-\beta+\alpha}^{2} L_{f} \varpi^{2}\left(\int_{-\infty}^{t} e^{-\delta(t-s)}(t-s)^{\beta-\alpha-1} d s\right)^{2} \sup _{t \in \mathbb{R}} E\|x(t)-\gamma(t)\|_{\alpha}^{2} \\
\leq & 4 M_{1-\beta+\alpha}^{2} L_{f} \varpi^{2} \delta^{2(\alpha-\beta)}[\Gamma(\beta-\alpha)]^{2} \sup _{t \in \mathbb{R}} E\|x(t)-\gamma(t)\|_{\alpha}^{2} .
\end{aligned}
$$

As regards the third term, we use again Cauchy-Schwarz inequality and obtain

$$
\begin{aligned}
& 4 E\left(\int_{-\infty}^{t}\left\|(-A)^{\alpha} T(t-s)\left[g\left(s, B_{2} x(s)\right)-g\left(s, B_{2} \gamma(s)\right)\right]\right\| d s\right)^{2} \\
\leq & 4 M_{\alpha}^{2} E\left(\int_{-\infty}^{t} e^{-\delta(t-s)}(t-s)^{-\alpha}\left\|g\left(s, B_{2} x(s)\right)-g\left(s, B_{2} \gamma(s)\right)\right\| d s\right)^{2} \\
\leq & 4 M_{\alpha}^{2} E\left[\left(\int_{-\infty}^{t} e^{-\delta(t-s)}(t-s)^{-\alpha} d s\right)\right. \\
& \left.\times\left(\int_{-\infty}^{t} e^{-\delta(t-s)}(t-s)^{-\alpha}\left\|g\left(s, B_{2} x(s)\right)-g\left(s, B_{2} \gamma(s)\right)\right\|^{2} d s\right)\right] \\
\leq & 4 M_{\alpha}^{2}\left(\int_{-\infty}^{t} e^{-\delta(t-s)}(t-s)^{-\alpha} d s\right) \\
& \times\left(\int_{-\infty}^{t} e^{-\delta(t-s)}(t-s)^{-\alpha} E\left\|g\left(s, B_{2} x(s)\right)-g\left(s, B_{2} \gamma(s)\right)\right\|^{2} d s\right) \\
\leq & 4 M_{\alpha}^{2} L_{g}\left(\int_{-\infty}^{t} e^{-\delta(t-s)}(t-s)^{-\alpha} d s\right) \\
& \times\left(\int_{-\infty}^{t} e^{-\delta(t-s)}(t-s)^{-\alpha} E\left\|B_{2} x(s)-B_{2} \gamma(s)\right\|^{2} d s\right) \\
\leq & 4 M_{\alpha}^{2} L_{g} \varpi^{2}\left(\int_{-\infty}^{t} e^{-\delta(t-s)}(t-s)^{-\alpha} d s\right)^{2} \sup _{t \in \mathbb{R}} E\|x(t)-\gamma(t)\|_{\alpha}^{2} \\
\leq & 4 M_{\alpha}^{2} L_{g} \varpi^{2} \delta^{2(\alpha-1)}[\Gamma(1-\alpha)]^{2} \sup _{t \in \mathbb{R}} E\|x(t)-\gamma(t)\|_{\alpha}^{2} .
\end{aligned}
$$

As far as the last term is concerned, by the Ito integral, we get

$$
\begin{aligned}
& 4 E\left\|\int_{-\infty}^{t} T(t-s)\left[h\left(s, B_{3} x(s)\right)-h\left(s, B_{3} \gamma(s)\right)\right] d W(s)\right\|_{\alpha}^{2} \\
\leq & 4 E\left(\int_{-\infty}^{t}\left\|(-A)^{\alpha} T(t-s)\left[h\left(s, B_{3} x(s)\right)-h\left(s, B_{3} \gamma(s)\right)\right]\right\|^{2} d s\right) \\
\leq & 4 M_{\alpha}^{2} \int_{-\infty}^{t} e^{-2 \delta(t-s)}(t-s)^{-2 \alpha} E\left\|h\left(s, B_{3} x(s)\right)-h\left(s, B_{3} \gamma(s)\right)\right\|^{2} d s \\
\leq & 4 M_{\alpha}^{2} L_{h} \int_{-\infty}^{t} e^{-2 \delta(t-s)}(t-s)^{-2 \alpha} E\left\|B_{3} x(s)-B_{3} \gamma(s)\right\|^{2} d s \\
\leq & 4 M_{\alpha}^{2} L_{h} \varpi^{2}\left(\int_{-\infty}^{t} e^{-2 \delta(t-s)}(t-s)^{-2 \alpha} d s\right) \sup _{t \in \mathbb{R}} E\|x(t)-\gamma(t)\|_{\alpha}^{2} \\
\leq & 4 M_{\alpha}^{2} L_{h} \varpi^{2}(2 \delta)^{2 \alpha-1} \Gamma(1-2 \alpha) \sup _{t \in \mathbb{R}} E\|x(t)-\gamma(t)\|_{\alpha}^{2} .
\end{aligned}
$$


Thus, by combining, it follows that, for each $t \in \mathbb{R}$,

$$
\begin{aligned}
& E\|(\Lambda x)(t)-(\Lambda y)(t)\|_{\alpha}^{2} \\
\leq & 4 \varpi^{2}\left\{\left\|(-A)^{\alpha-\beta}\right\|^{2} L_{f}+M_{1-\beta+\alpha}^{2} \delta^{2(\alpha-\beta)}[\Gamma(\beta-\alpha)]^{2} L_{f}+M_{\alpha}^{2} \delta^{2(\alpha-1)}[\Gamma(1-\alpha)]^{2} L_{g}\right. \\
& \left.+M_{\alpha}^{2} L_{h}(2 \delta)^{2 \alpha-1} \Gamma(1-2 \alpha)\right\} \sup _{t \in \mathbb{R}} E\|x(t)-\gamma(t)\|_{\alpha^{\prime}}^{2}
\end{aligned}
$$

that is,

$$
\|(\Lambda x)(t)-(\Lambda y)(t)\|_{2, \alpha}^{2} \leq L_{0} \sup _{t \in \mathbb{R}}\|x(t)-\gamma(t)\|_{2, \alpha}^{2} .
$$

Note that

$$
\sup _{t \in \mathbb{R}}\|x(t)-\gamma(t)\|_{2, \alpha}^{2} \leq\left(\sup _{t \in \mathbb{R}}\|x(t)-\gamma(t)\|_{2, \alpha}\right)^{2},
$$

and (3.5) together with (3.6) gives, for each $t \in \mathbb{R}$,

$$
\|(\Lambda x)(t)-(\Lambda y)(t)\|_{2, \alpha} \leq \sqrt{L_{0}}\|x-y\|_{\infty, \alpha} .
$$

Hence, we obtain

$$
\|\Lambda x-\Lambda y\|_{\infty, \alpha}=\sup _{t \in \mathbb{R}}\|(\Lambda x)(t)-(\Lambda y)(t)\|_{2, \alpha} \leq \sqrt{L_{0}}\|x-y\|_{\infty, \alpha} .
$$

which implies that $\Lambda$ is a contraction by (3.1). Therefore, by the Banach contraction principle, we conclude that there exists a unique fixed point $x(\cdot)$ for $\Lambda$ in $A A\left(\mathbb{R} ; L^{2}\left(\mathbb{P}, \mathbb{H}_{\alpha}\right)\right)$, such that $\Lambda x=x$, that is

$$
\begin{aligned}
x(t)= & f\left(t, B_{1} x(t)\right)+\int_{-\infty}^{t} A T(t-s) f\left(s, B_{1} x(s)\right) d s \\
& +\int_{-\infty}^{t} T(t-s) g\left(s, B_{2} x(s)\right) d s+\int_{-\infty}^{t} T(t-s) h\left(s, B_{3} x(s)\right) d W(s)
\end{aligned}
$$

for all $t \in \mathbb{R}$. If we let $x(a)=f\left(a, B_{1} x(a)\right)+\int_{-\infty}^{a} A T(a-s) f\left(s, B_{1} x(s)\right) d s+\int_{-\infty}^{a} T(a-s) g\left(s, B_{2} x(s)\right) d s+\int_{-\infty}^{a} T(a-s) h\left(s, B_{3} x(s)\right) d W(s)$, then

$$
\begin{aligned}
T(t-a) x(a)= & T(t-a) f\left(a, B_{1} x(a)\right)+\int_{-\infty}^{a} A T(t-s) f\left(s, B_{1} x(s)\right) d s \\
& +\int_{-\infty}^{a} T(t-s) g\left(s, B_{2} x(s)\right) d s+\int_{-\infty}^{a} T(t-s) h\left(s, B_{3} x(s)\right) d W(s) .
\end{aligned}
$$

However, for $t \geq a$,

$$
\begin{aligned}
& \int_{a}^{t} T(t-s) h\left(s, B_{3} x(s)\right) d W(s) \\
= & \int_{-\infty}^{t} T(t-s) h\left(s, B_{3} x(s)\right) d W(s)-\int_{-\infty}^{t} T(t-s) h\left(s, B_{3} x(s)\right) d W(s) \\
= & x(t)-f\left(t, B_{1} x(t)\right)-\int_{-\infty}^{t} A T(t-s) f\left(s, B_{1} x(s)\right) d s-\int_{-\infty}^{t} T(t-s) g\left(s, B_{2} x(s)\right) d s \\
& -T(t-a)\left[x(a)-f\left(a, B_{1} x(a)\right)\right] \\
& +\int_{-\infty}^{a} A T(t-s) f\left(s, B_{1} x(s)\right) d s+\int_{-\infty}^{a} T(t-s) g\left(s, B_{2} x(s)\right) d s \\
= & x(t)-T(t-a)\left[x(a)-f\left(a, B_{1} x(a)\right)\right]-f\left(t, B_{1} x(t)\right) \\
& -\int_{-\infty}^{t} A T(t-s) f\left(s, B_{1} x(s)\right) d s-\int_{-\infty}^{t} T(t-s) g\left(s, B_{2} x(s)\right) d s .
\end{aligned}
$$


In conclusion, $\left.x(t)=T(t-a) x x(a)-f\left(a, B_{1} x(a)\right)\right]+f\left(t, B_{1} x(t)\right)+\int_{a}^{t} A T(t-s) f\left(s, B_{1} x(s)\right) d s+\int_{a}^{t} T(t-s) g\left(s, B_{2} x(s)\right) d s+\int_{a}^{t} T(t-s) h\left(s, B_{3} x(s)\right) d W(s)$ is a mild solution of equation (1.1) and $x(\cdot) \in A A\left(\mathbb{R} ; L^{2}\left(\mathbb{P}, H_{\alpha}\right)\right)$. The proof is completed.

Remark 3.1 The results of Theorem 3.1 can be used to study the existence and uniqueness of square-mean almost automorphic mild solutions to the example in [18].

\begin{abstract}
Acknowledgements
The authors are grateful to the anonymous referees for their valuable comments and suggestions to improve this paper. This study was supported by NNSF of China (10901075), Program for New Century Excellent Talents in University (NCET-10-0022), the Key Project of Chinese Ministry of Education (210226), the Scientific Research Fund of Gansu Provincial Education Department (0804-08), and Qing Lan Talent Engineering Funds (QL-05-16A) from Lanzhou Jiaotong University.
\end{abstract}

\title{
Author details
}

'Department of Mathematics, Lanzhou Jiaotong University, Lanzhou, Gansu 730070, PR China ${ }^{2}$ Department of Mathematics, Morgan State University, 1700 E. Cold Spring Lane, Baltimore, MD 21251, USA

\section{Authors' contributions}

YKC carried out the main proof of this manuscript, ZHZ drafted the manuscript and corrected the main theorems, GMN gave two lemmas, corrected the main theorems and improved this manuscript.

\section{Competing interests}

The authors declare that they have no competing interests.

Received: 20 September 2010 Accepted: 14 June 2011 Published: 14 June 2011

\section{References}

1. Bochner S: A new approach to almost automorphicity. Proc Natl Acad Sci USA 1962, 48:2039-2043.

2. Bochner S: Continuous mappings of almost automorphic and almost periodic functions. Proc Natl Acad Sci USA 1964, 52:907-910.

3. N'Guérékata GM: Almost Automorphic and Almost Periodic Functions in Abstract Space. Kluwer Academic Plenum Publishers, New York; 2001.

4. N'Guérékata GM: Topics in Almost Automorphy. Springer, New York; 2005.

5. Abbas S, Bahuguna D: Almost periodic solutions of neutral functional differential equations. Comput Math Appl 2008, 55:2593-2601.

6. Diagana T, N'Guérékata GM: Almost automorphic solutions to semilinear evolution equations. Funct Differential Equation 2006, 13:195-206.

7. Diagana T, N'Guérékata GM: Almost automorphic solutions to some classes of partial evolution equations. Appl Math Lett 2007, 20:462-466.

8. Ding H-S, Long W, N'Guérékata GM: Almost automorphic solutions of nonautonomous evolution equations. Nonlinear Anal TMA 2010, 70:4158-4164.

9. Ding H-S, Long W, N'Guérékata GM: A composition theorem for weighted pseudo almost automorphic functions and applications. Nonlinear Anal TMA 2010, 73:2644-2650

10. Ding H-S, Liang J, Xiao T-J: Almost automorphic solutions to nonautonomous semilinear evolution equations in Banach spaces. Nonlinear Anal TMA 2010, 73:1426-1438.

11. Hernández E, Henríquez HR: Existence of periodic solutions of partial neutral functional differential equations with unbounded delay. J Math Anal Appl 1998, 221:499-522.

12. Henríquez $\mathrm{HR}$, Vasquez $\mathrm{CH}$ : Almost periodic solutions of abstract retarded functional differential equations with unbounded delay. Acta Appl Math 1999, 57:105-132.

13. N'Guérékata GM: Existence and uniqueness of almost automorphic mild solutions to some semilinear abstract differential equations. Semigroup Forum 2004, 69:80-86.

14. Zhao Z-H, Chang Y-K, Nieto JJ: Almost automorphic and pseudo almost automorphic mild solutions to an abstract differential equation in Banach spaces. Nonlinear Anal TMA 2010, 72:1886-1894.

15. Zhao Z-H, Chang Y-K, Li W-S: Asymptotically almost periodic, almost periodic and pseudo almost periodic mild solutions for neutral differential equations. Nonlinear Anal RWA 2010, 11:3037-3044.

16. Bezandry P, Diagana T: Existence of almost periodic solutions to some stochastic differential equations. Appl Anal 2007, 86:819-827.

17. Bezandry P: Existence of almost periodic solutions to some functional integro-differential stochastic evolution equations. Stat Prob Lett 2008, 78:2844-2849.

18. Bezandry P, Diagana T: Existence of quadratic-mean almost periodic solutions to some stochastic hyperbolic differential equations. Electron J Differential Equations 2009, 111:1-14.

19. Da Prato G, Tudor C: Periodic and almost periodic solutions for semilinear stochastic evolution equations. Stoch Anal Appl 1995, 13:13-33.

20. Tudor CA: Almost periodic solutions of affine stochastic evolutions equations. Stoch Stoch Rep 1992, 38:251-266.

21. Tudor CA, Tudor M: Pseudo almost periodic solutions of some stochastic differential equations. Math Rep (Bucur.) 1999, 1:305-314

22. Dorogovtsev AYa, Ortega OA: On the existence of periodic solutions of a stochastic equation in a Hilbert space. Visnik Kiiv Univ Ser Math Mekh 1988, 115(30):21-30. 
23. Fu MM, Liu ZX: Square-mean almost automorphic solutions for some stochastic differential equations. Proc Am Math Soc 2010, 138:3689-3701.

24. Da Prato G, Zabczyk J: Stochastic Equations in Infinite Dimensions. Cambridge University Press, Cambridge; 1992.

25. Pazy A: Semigroups of Linear Operators and Applications to Partial Equations. In Applied Mathematical Sciences. Volume 44. Springer-Verlag, New York; 1983.

26. Ichikawa A: Stability of semilinear stochastic evolution equations. J Math Anal App/ 1982, 90:12-44.

doi:10.1186/1687-1847-2011-9

Cite this article as: Chang et al:: Square-mean almost automorphic mild solutions to some stochastic differential equations in a Hilbert space. Advances in Difference Equations 2011 2011:9.

Submit your manuscript to a SpringerOpen ${ }^{\circ}$ journal and benefit from:

- Convenient online submission

- Rigorous peer review

- Immediate publication on acceptance

- Open access: articles freely available online

- High visibility within the field

- Retaining the copyright to your article

Submit your next manuscript at $\boldsymbol{s p r i n g e r o p e n . c o m ~}$ 